\title{
The Design of social security information management system
}

\author{
Cao Wanli \\ Department of Management, China West Normal University, Nanchong 637000, China \\ Ccyy789@sohu.com
}

\begin{abstract}
The social security information management system based on three-tier model and the client-server framework is built, which in close connection with social security archives information. At the same time, the amount of data, namely the underlying architecture code design, system architecture design, and resolve client desktop three levels to form. The social security system has the formation of the window, which informs retrieval of data management applications for comprehensive
\end{abstract}

Keywords-Social security; information management system; client-server schema; the design and operation.

\section{INTRODUCTION}

Client / Server have large data storage capacity, the effectiveness of operational as well as a wide interface interaction and many other advantages, along with the overall size of the social security industry operators expanding steadily optimization and comprehensive quality, maintain operational data management support social security industry systems were quickly showing a new trend of intelligent, portable intensive nature. This article is based on the three-Client / Server information management system as a social security infrastructure, combined with the Intranet network rollout, the interaction of these three components and client components, application servers, and database servers by connecting adapter, the final formation of the information management system to build anew intensive social security[1].

\section{THREE - LAYER CLIENT-SERVER OVERALL OVERVIEW}

2.1 The connotation features of the three-tier client/server architecture

Three-tier Client / Server benefits information management system in the computerized platform

under the basic principle of operation is as follows: First, the technical operations staff paper social security information through computer software assisted into electronic document format, generally include the suffix named xls, ppt, doc three formats file, then upload stored in the database server as a backup when you need to call to see than the required information when permissions by client window command operation command is given, and issued a call instruction is transmitted to the application, the application further database request data read and write permissions, the database server in response to its request, will request the data transmission to the application program, then the data transfer application to the client, the client window in the form of shows to the user personnel, and ultimately complete information data look up. Social security co-ordination of information management is the basis of the complexity of the frequency of operation of the instruction, the three-tier Client / Server with integrated, convenient, interactive technology is basically to meet a series of data storage, calls to modify[1-3].

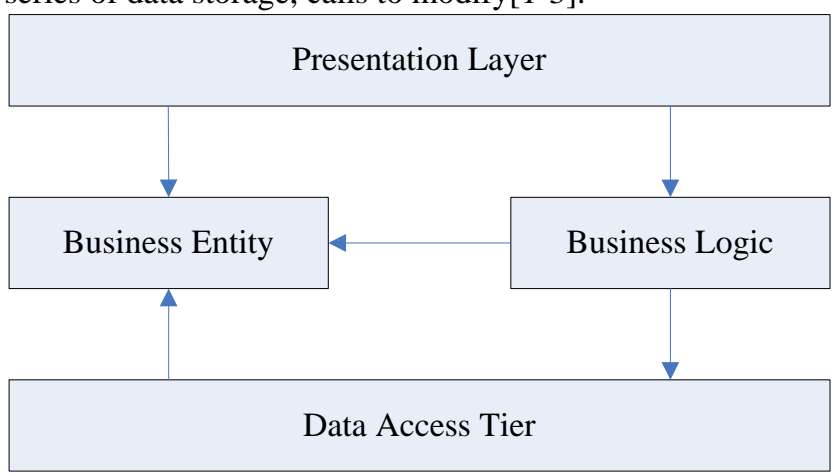

Fig.1 Three layer Client/Server model

2.2 The advantages of the three-tier client / server architecture

First, the application server running the data load is lighter. The simplest C/S architecture database application consists of two parts, the client application and the database server program. Both are called the foreground programs and background programs. Machine running the database server program, also known as the application server. Once the server is started, you wait for the response to the client request at anytime; client application running on the user's own computer, corresponding to the database server, called the client computer, when the need to carry out any operation on the data in the database, the client program will automatically find a server program, and request, the server program to make a response based on a predetermined rule, return the result, application server running data lighter load.

Second, the data storage management functions more transparent. Database applications, data storage management functions by the server program and the client application independently conducted, foreground application may be in violation of the rules, and usually those different (whether known or unknown) operating data, not concentrated in the server program to achieve, such as the visitor's permission, the number can be repeated, must have customers to establish order rules. All of these, for the end-user of the work on the foreground program is "transparent", they do not have to intervene (usually can not interfere in) behind the process, you can do the all the work. Ina client-server 
architecture, the foreground program is not very "thin" troublesome thing to the server and network. In C/S system, the database can not truly become public, professional warehouse by independent specialized management [4].

$\mathrm{C} / \mathrm{S}$ architecture disadvantage is the high maintenance costs and the large investment. First, the use of the $\mathrm{C} / \mathrm{S}$ structure, to select the appropriate database platform database data to achieve true "unified" data synchronization by the database system to manage the distribution of the two, but both logic operation directly access the same database in order to achieve effective, they are the problems, if you need to create a "real-time" data synchronization, you must be established between the two real-time communication connections to maintain both the database server to run online network management staff necessary to maintenance and management of server maintenance and management, but also on the client, which requires high investment and complex technical support, and maintenance costs are very high, the large amount of maintenance tasks. Second, traditional C/S structure software need to develop different versions of software for different operating systems, no longer meet the work requirements due to the upgrading of the product is very costly and inefficient. After the emergence of such across-platform language in JAVA, B/S architecture is more severe attacks on $\mathrm{C} / \mathrm{S}$, and the formation of the threats and challenges.

\section{THE SOCIAL SECURITY DIVISION OF ARCHIVES \\ INFORMATION MANAGEMENT SYSTEM DESIGN AND THE STRUCTURE OF NODES}

3.1 The overall structure of the system partition

Object of social security information management is a relatively complicated process many crushing operation, and each object corresponding to the process operation is basically to be involved in data archiving, modify the attributes, permissions limited formatting basic planning, also need a computer platform top of the completion of such Filing, additions and deletions, modifications, borrow, inspection, print, change, statistics and so trivial repeated the details of the operation. In view of this, the level of the overall system defining, category partition, and subheading Ordering is necessary and critical, we can through the use of a parent directory, subdirectory, under the three directories and subdirectories basic form the overall structure of the system is divided, wherein the parent the main keywords directory for the social security of the subdirectory for the main keywords related extended phrase, three directories are mainly responsible for phrases update this whole structure is divided is clear, orderly.

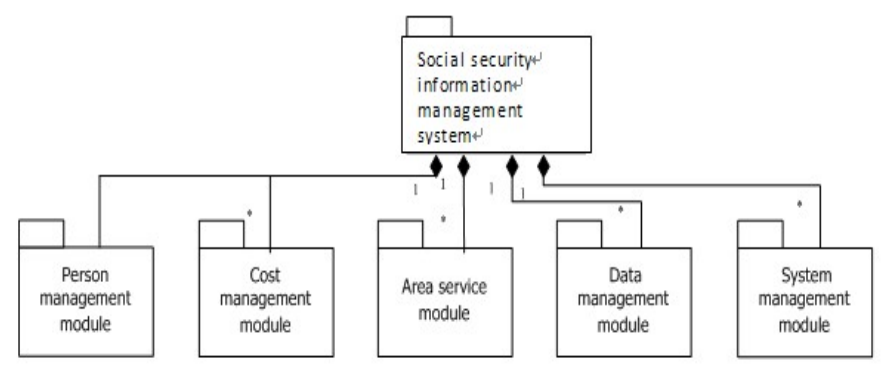

Fig. 2 Function module chart of Social security information management system

Is related operators conducting divided when the structure of the overall system, be sure to focus intensive treatment for the level of detail, more extensive and complex field of social security information covered includes not only such as medical and health services, cultural and educational services, labor and employment basic services, residential services, the lonely old residues young service, disability rehabilitation services, crime prevention and probation services, mental health services, public welfare services. And differentiation along with the values of social groups change their clients not to be limited to groups of objects under the laws and regulations of the state organs, and extend gradually expand to the elderly, the disabled, women, children, young people, soldiers and their families, poverty as well as other needy members of society and family and so on. Form of social security services, presented the basic auxiliary, human, material and financial resources of the state, collectives and individuals to set up the adoption of social security, community services, family services, casework, group services is rising rapidly in recent years. These new stage elements have a higher demand for social security information system set up to build, especially for the division of the structure of the overall system, becomes all the more necessary and crucial. In addition to the parent directory, subdirectory, and subdirectories consists of three directories form the structure of the overall system is divided addition to the technical operations personnel also need to continue to refine the classification of these new elements of the new content directory set through the built-in LAN link, for example, the new elements of the new file pointing to the other server terminal further while a wealth of information on reserves, effectively protecting personnel for social security information inquiries, call and to download.

3.2 The realization of social security information management system

I believe that the expansion based on the three-tier Client / Server architecture, we can use the following three configurations for specific operations: The first is an external server set up as a data layer, functional layer data buffer for complex information management system of social security, so not only to enhance the effectiveness of the exchange of data between the data layer, functional layer, but also save the cost of related hardware and software resources invested; followed were erected two servers as a data layer, functional layer data exchange source, such erection mode high mobility, but the need for frequent exchange of data and 
produce heat-power, is not conducive to the performance and security of server hardware; third is a direct function of layer placed above the client component, the Although the mode to facilitate maintenance troubleshooting and performance, but for the heavy load of the client, very easy to cause physical injury. Based on the above three kinds of the erected mode of pros and cons, and combined with the effectiveness of the benefits of information management, I believe that appropriate to select the second mode is the most economical and reasonable [5].

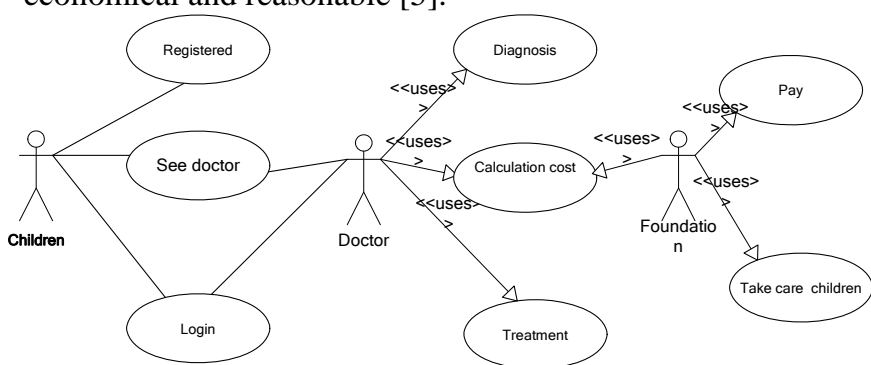

Fig. 3 Children see doctor displayed by use case diagram.

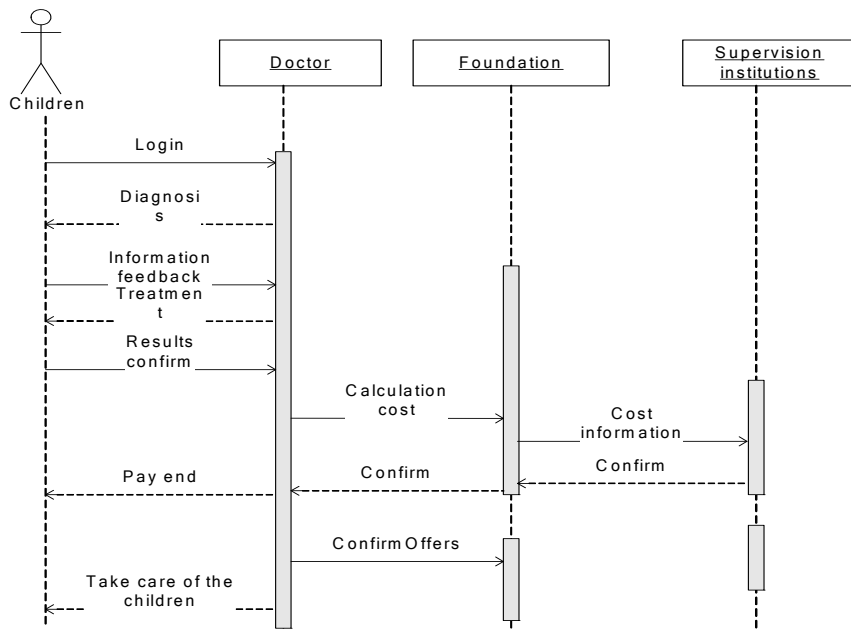

Fig. 4 Children see doctor sequence diagram

Advantage of the erection of two servers as a data layer, functional layer data exchange source information data exchanged frequent high effectiveness, throughput and information management system for the content level complex, a huge amount of information on social security words, does have a significant obvious assisted adjustment utility. As above said, frequently exchanged due to the amount of data, so that the effectiveness of the heat generated under the entire system operation is quite large, so that the two servers are in the trend of the load operation for a long period of time under, for the entire information management system. the security and stability of the great constraints burdens.

3.3 Database Design of dynamic linking

Similar to the basic architecture of the benefits of information management systems and other information management systems, data server as the basis for information exchange medium, the more common database server mode was undoubtedly the SQLServer2005. The database principle of operation is simple and convenient operation, easy maintenance, not only can perform table design view sketch, flip-flop design based on the storage limit process, and for the contents of each process are set flexibly add data options. For example, when the table during table design, we can category, the data for each table corresponding fill, and set the format of its type, access permissions, check the password, etc., not only greatly enhance the effectiveness of the information management of data manipulation and strong guarantee for the security of information and data.

Dynamic linking, the executable file is loaded or run by the operating system loader to load libraries. Most operating systems will resolve external references (such as libraries)as part of the loading process. In these systems, the executable file contains a table called import directory, each item of the table contains the name of a library. Recorded in the table name, search the library of the loader on the hard disk, and then load it into memory pre uncertain position, and after to update executable program based on the address of the library to determine load library. Executable program library based on updated information call the library function or data in the reference library. This type of dynamically loaded when most systems, including Windows and Linux adoption. Loader to load the application software to complete one of the most complex work is loaded when the link ${ }^{[6]}$.

Other operating systems may be resolved at run time references. In these systems, the executable program calls the operating system API, the name of the library, with the number and function of the parameters of the function in the library pass. The operating system is responsible for parsing and on behalf of the application calls the appropriate function. This dynamic link called run-time link. Runtime overhead for each call will link to be much slower and have a negative impact on the performance of the application. Modern operating systems have rarely used the runtime linker.

\section{SOCIAL SECURITY INFORMATION MANAGEMENT PROCESSES TO PERFORM AN ACTION LINK}

\subsection{Windows desktop client operations}

The Desktop client information management system of social security under the three-tier Client / Server architecture, window-type operation is the most personalized and convenient, a series of day-to-day operations, such as search query, print, modify, delete Browse lend summary statistics handover, destroyed emptied, etc. can be real-time dynamic window operation successfully completed, will undoubtedly have a convenient and efficient for the large amount of information processing, the operation is time-consuming, the implementation of the high strength of the social security information management, the practical benefits of saving time and effort. 


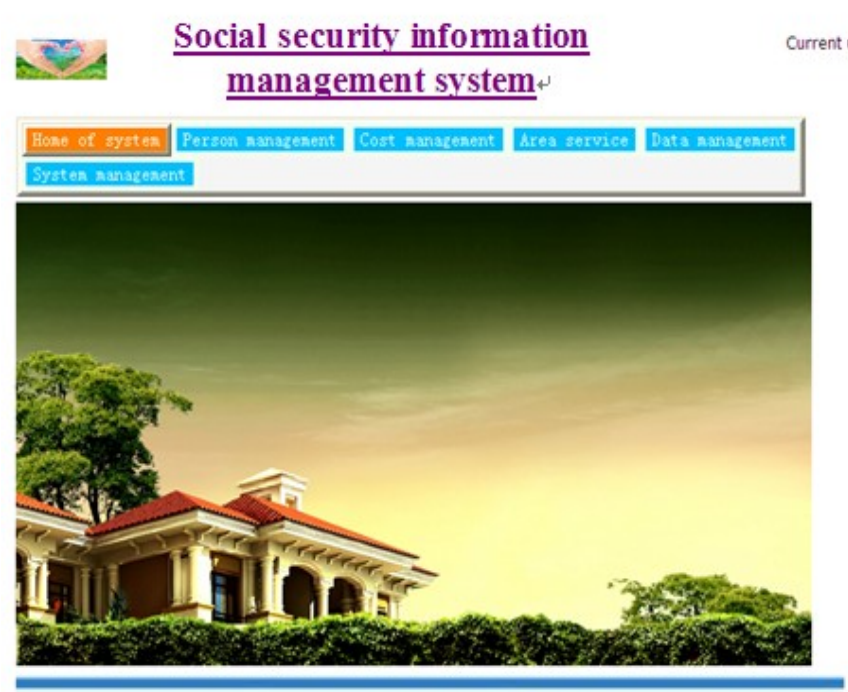

All rights reserved (2010-2015)

Fig. 5 The main interface of the Social security information management system

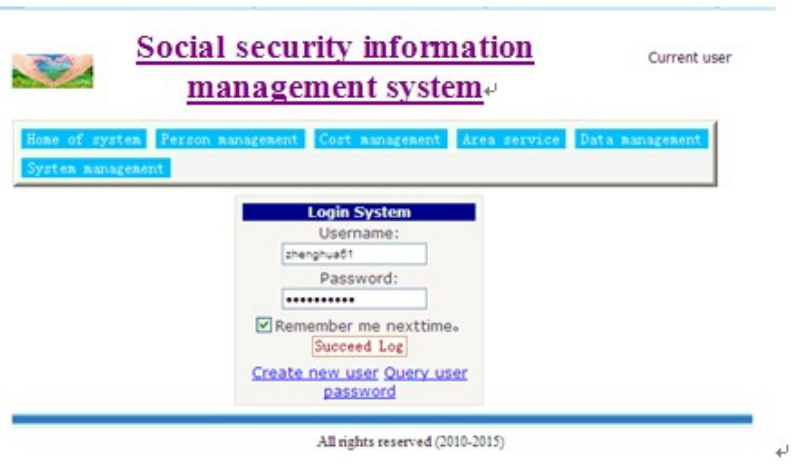

Fig. 6 Login interface of social security information management system

4.2 Set up application server software layer to form

The basic role of the three-tier Client / Server architecture, the application software layer server set up to set up appears to be more flexible and diverse, and based on the convenience and effectiveness of information management system of social security and intensive comprehensive requirements I believe that can be achieved by creating aActiveX.DLL well ActiveX EXE dynamic link library files, and executable interactive interface mode of operation. This model can not only handed over the package database interface package file table. The table of contents set custom human diversity and specific information, based on actual data; such fully and effectively enhance the flexibility to expand the social security information management systems perform.

\section{CONCLUSION}

Computer information management in information suitable for application in the management of social security as a component of the long-term, system- wideprogram works, in the social security sector play an update operation to improve thesystem key utility. Further ways of improving the widening of the computer informationmanagement system to build channels, the in-depth development of professional skillstraining of relevant personnel management of computer technology and application of real time detailed operation, so as to give full play to this " Blade of Science andTechnology " unique advantage, and ultimately social security continued, coordinated andrapid development of information management system to promote.

\section{REFERENCES}

[1] WANG Wen-fa; MA Yan; LI Hong-da. Four-tier structure based on.NET and its application in integrated information system[J]. Computer Engineering and Design, 2009, 30 (4): 912-915.

[2] LI B., CHANG D. H., LIANG H. Research in Data Model of Geographical Database Engine[J]. Journal of Zheng zhou Institute of Surveying and Mapping. 2006, 123(5):363-365.

[3] Wang Ming; He Feng; Wang Hui. Research of Integration Platform Facing to Three-tier Structure Information System[J]. Microcomputer Applications, 2011, 27(3): 23-26.

[4] Wang Zhaobin. Design of student management information system [J]. Network leader online education, 2012 (8).

[5] Xiong ShuiPing. Information management system design based on ExtJS [J]. Science, 2011 (11).

[6] Lin Bin. Based on enterprise information management system design and realization of networked environment $[\mathrm{J}]$. Science and technology science and technology world 2011 (7). 